\title{
BMJ Open Postpartum urinary tract infection by mode of delivery: a Danish nationwide cohort study
}

\author{
Tina Djernis Gundersen, ${ }^{1}$ Lone Krebs, ${ }^{2}$ Ellen Christine Leth Loekkegaard, ${ }^{1}$ \\ Steen Christian Rasmussen, ${ }^{3}$ Julie Glavind, ${ }^{4}$ Tine Dalsgaard Clausen ${ }^{1}$
}

To cite: Gundersen TD, Krebs L, Loekkegaard ECL, et al. Postpartum urinary tract infection by mode of delivery: a Danish nationwide cohort study. BMJ Open 2018;8:e018479. doi:10.1136/ bmjopen-2017-018479

- Prepublication history and additional material for this paper are available online. To view these files, please visit the journal online (http://dx.doi. org/10.1136/bmjopen-2017018479).

Received 3 July 2017

Revised 27 January 2018

Accepted 9 February 2018

Check for updates

${ }^{1}$ Department of Gynecology and Obstetrics, Nordsjaellands Hospital, Hilleroed, Denmark ${ }^{2}$ Department of Gynecology and Obstetrics, Holbaek Sygehus, Holbaek, Denmark

${ }^{3}$ Department of Clinical

Microbiology, Hvidovre Hospital, Hvidovre, Denmark

${ }^{4}$ Institute for Clinical Medicine, Department of Gynecology and Obstetrics, Aarhus University Hospital, Aarhus, Denmark

Correspondence to

Ms. Tina Djernis Gundersen; tinadgundersen@gmail.com

\section{ABSTRACT}

Objectives To examine the association between postpartum urinary tract infection and intended mode of delivery as well as actual mode of delivery.

Design Retrospective cohort study.

Setting and participants All live births in Denmark between 2004 and 2010 ( $n=450$ 856). Births were classified by intended caesarean delivery $(n=45053)$ or intended vaginal delivery $(n=405803)$, and by actual mode of delivery: spontaneous vaginal delivery, operative vaginal delivery, emergency or planned caesarean delivery in labour or prelabour.

Primary and secondary outcome measures The primary outcome measure was postpartum urinary tract infection ( $n=16295)$ within 30 days post partum, defined as either a diagnosis of urinary tract infection in the National Patient Registry or redemption of urinary tract infection-specific antibiotics recorded in the Register of Medicinal Product Statistics.

Results We found that $4.6 \%$ of women with intended caesarean delivery and $3.5 \%$ of women with intended vaginal delivery were treated for postpartum urinary tract infection. Women with intended caesarean delivery had a significantly increased risk of postpartum urinary tract infection compared with women with intended vaginal delivery (OR 1.33, 95\% $\mathrm{Cl} 1.27$ to 1.40$)$, after adjustment for age at delivery, smoking, body mass index, educational level, gestational diabetes mellitus, infection during pregnancy, birth weight, preterm delivery, preterm prelabour rupture of membranes, pre-eclampsia, parity and previous caesarean delivery (adjusted OR 1.24, $95 \% \mathrm{Cl} 1.17$ to 1.46 ). Using actual mode of delivery as exposure, all types of operative delivery had an equally increased risk of postpartum urinary tract infection compared with spontaneous vaginal delivery. Conclusions Compared with intended vaginal delivery, intended caesarean delivery was significantly associated with a higher risk of postpartum urinary tract infection. Future studies should focus on reducing routine catheterisation prior to operative vaginal delivery as well as improving procedures related to catheterisation.

\section{INTRODUCTION}

Urinary tract infection (UTI) is a common postpartum infection occurring in 2\%-4\% of all deliveries. ${ }^{1-3}$ Although postpartum UTI is usually a mild infection, it is associated

\section{Strengths and limitations of this study}

- Large nationwide study including data from the Danish birth cohort $(n=450856$ )

- High-quality data where prospective collection limits the risk of selection and information bias.

- Evaluates the risk of urinary tract infection (UTI) by intended as well as actual mode of delivery.

- The diagnosis of postpartum UTI was not confirmed by urinary cultures.

Cohort study without the ability to assess causality.

with discomfort, prolonged hospital stay and readmission and has been associated with an increased risk of discontinued breast feeding. ${ }^{1} 4$ In Denmark and many other countries, most women deliver in hospitals and are discharged a few hours after giving birth, ${ }^{156}$ and $79 \%$ of all postpartum UTIs in Denmark will occur after discharge and are diagnosed and treated by a general practitioner. $^{36}$

Especially, when counselling women aiming for caesarean delivery, on maternal request without obstetric indications, it is essential to provide information regarding both major and minor complications. In several studies, the risk of postpartum UTI and other puerperal infections has been found to be increased after caesarean delivery as compared with vaginal delivery. ${ }^{5-7}$ However, there is a paucity of data evaluating the risk of postpartum UTI by intended mode of delivery. This information is useful in the clinical setting when counselling the pregnant woman prior to delivery about the perceived risks attributed to the various modes of delivery. ${ }^{8}$ As the prevalence of emergency caesarean delivery or operative vaginal delivery among women with intended vaginal delivery varies in different populations, information regarding risks attributed to the actual mode of delivery is also important when using data from other populations. 
We examined the risk of postpartum UTI by intended and actual mode of delivery in a Danish national birth cohort.

\section{MATERIALS AND METHODS}

This study is a nationwide, register-based cohort study of all live births in Denmark from 1 January 2004 to 31 December 2010 ( $n=450856)$.

A database was established based on four population-based Danish registers: the Medical Birth Registry, ${ }^{9}$ the Fertility Database,${ }^{10}$ the National Patient Registry, ${ }^{11}$ the Register of Medicinal Product Statistics ${ }^{12}$ and additional data from Statistics Denmark. ${ }^{13}$

No international review board approval was needed for this cohort study in accordance with Danish regulations. By use of the civil registration number, which is a unique personal identification number, linkage between registers was possible. ${ }^{13}$ After linkage, data were de-identified to ensure data safety.

The Medical Birth Registry provided data on date of birth, vital status at birth, mode of delivery, parity, multiple gestation, maternal smoking status, body mass index (BMI), previous caesarean delivery, birth weight and gestational age at birth. The Fertility Database linked children to their mothers and provided date of birth for the mother. The National Patient Registry contributed with hospital admission dates, discharge diagnoses for complications during pregnancy and codes for surgical procedures related to delivery. The Register of Medicinal Product Statistics provided data on individual redemption of prescriptions for antibiotics, all of which require a prescription. When a prescription is redeemed, the buyer's civil registration number and the Anatomical Therapeutic Chemical code of the drug prescribed are automatically linked to the specific date. Statistics Denmark provided information on maternal educational status.

\section{Variable definitions \\ Outcome}

The primary outcome was defined as a hospital discharge diagnosis of lower or upper UTI or redemption of a prescription for a UTI-specific antibiotic within 30 days post partum or both. UTI-specific antibiotics were defined as antibiotics used almost exclusively to treat UTI in monotherapy, in accordance with regional and national guidelines (sulfamethizole (J01EB02), mecillinam (J01CA11), nitrofurantoin (J01XE01), trimethoprim (J01EE01), amoxicillin (J01CA04), pivampicillin (J01CA02), ampicillin (J01CA01) and no concurrent treatment with metronidazole (P01AB01)). ${ }^{14}{ }^{15}$ To prevent inclusion of women, treated for other infections than UTI, women who simultaneously redeemed a UTI-specific antibiotic and a metronidazole prescription were considered as having endometritis and they were therefore classified as not having a postpartum UTI (online supplementary appendix). For sensitivity analyses, we applied a more restrictive definition of UTI. UTI was defined by a hospital discharge diagnosis of lower or upper UTI or redemption of a prescription for a UTI-specific antibiotic within 30 days post partum, however, women who at any timepoint within the first 30 days post partum redeemed a prescription for dicloxacillin, benzylpenicillin or metronidazole (antibiotics not used for UTI treatment) were classified as not having UTI, as we considered that they had been treated for another kind of infection, that erroneously had been diagnosed as a UTI (online supplementary appendix).

\section{Exposure}

Codes from the Nordic Medico-Statistical Committee classification of surgical procedures ${ }^{16}$ were used to define mode of delivery as: planned caesarean delivery prelabour, planned caesarean delivery in labour, emergency caesarean delivery prelabour, emergency caesarean delivery in labour and operative vaginal delivery. According to Danish guidelines, a caesarean delivery is classified as planned if the decision to deliver by caesarean delivery is made more than 8 hours before delivery.

The primary exposure was intended mode of delivery. Planned caesarean delivery prelabour and planned caesarean delivery in labour were merged to define intended caesarean delivery. Intended vaginal delivery included spontaneous or operative vaginal delivery and emergency caesarean delivery (prelabour or in labour). Spontaneous vaginal delivery was defined as delivery with no code recorded for an operative procedure at birth (online supplementary appendix). Secondary exposure was actual mode of delivery.

\section{Other covariates}

Covariates were selected based on theory and knowledge from previous studies. ${ }^{1617}$ Maternal covariates were age at delivery (continuous, years), smoking (current smoker, yes or no), pregestational BMI ( $\geq 25$ or $<25 \mathrm{~kg}$ / $\mathrm{m}^{2}$ ), educational level (measured as highest completed education at childbirth: elementary school/high school, short education/skilled worker, medium/long education) and previous caesarean delivery (yes or no). Perinatal covariates were gestational diabetes mellitus (GDM, yes or no), infection during pregnancy (infection-related diagnosis codes in pregnancy, yes or no), preterm birth ( $<37$ weeks gestation, yes or no), preterm prelabour rupture of membranes (PPROM, yes or no), pre-eclampsia (yes or no), parity (parous or primiparous), multiple gestation (yes or no) and child-related covariates were low birth weight ( $2500 \mathrm{~g}$, yes or no) (online supplementary appendix).

\section{Statistical analysis}

Background characteristics according to mode of delivery were calculated and compared using a $\chi^{2}$ test or Student's t-test, where appropriate. Logistic regression analyses were performed to evaluate the risk of postpartum UTI by 
mode of delivery and reported as crude ORs and adjusted ORs (aORs) with 95\% CIs. Missing values were handled by complete case analyses.

We defined two adjusted models for the analyses. In the first model, we adjusted for age at delivery, smoking, BMI, educational level, GDM, infection during pregnancy, low birth weight, preterm birth, PPROM, pre-eclampsia, parity and previous caesarean delivery. In the second model, we additionally included an interaction term between previous caesarean delivery and mode of delivery. When a significant interaction term was found, the effect was evaluated by stratified analyses. To evaluate the effect of previous caesarean delivery on the risk of postpartum UTI, stratified analyses among women with and without previous caesarean delivery were performed.

We further repeated the main analyses with a 14-day and a 45-day postpartum follow-up to examine whether mode of delivery affected the timing of postpartum UTI. Sensitivity analyses were performed on hospital admissions and redemption for antibiotic prescriptions separately and using the restrictive definition of postpartum UTI.

Statistical analyses were performed using Rstudio V.3.2.3. $\mathrm{P}<0.05$ was considered statistically significant.
RESULTS

We included all live births $(\mathrm{n}=450856)$ from 1 January 2004 to 31 December 2010, $45053(10 \%)$ of which were intended caesarean deliveries, $53583(11.9 \%)$ were emergency caesarean deliveries and $33792(7.5 \%)$ operative vaginal deliveries. Most demographic and delivery characteristics differed by intended mode of delivery (table 1 ). Background characteristics by actual mode of delivery are shown in online supplementary table 1.

\section{Risk of postpartum UTI by intended mode of delivery}

Among women with intended caesarean delivery, 4.6\% had a postpartum UTI, 0.2 percentage points of which had a hospital admission with postpartum UTI and $4.5 \%$ redeemed a prescription for UTI-specific antibiotics. In women with intended vaginal delivery, 3.5\% were diagnosed with postpartum UTI, $0.1 \%$ of which were treated in hospital and $3.4 \%$ redeemed a prescription for UTI-specific antibiotics within 30 days after delivery (figure 1). In crude analysis, the risk of postpartum UTI was significantly increased in women with intended caesarean delivery compared with intended vaginal delivery (OR 1.33, 95\% CI 1.27 to 1.40 ), and the risk persisted increased after adjustment for all covariates (aOR 1.36, 95\% CI 1.27 to 1.46 ) (table 2).

Table 1 Maternal and perinatal characteristics in deliveries including live-born children in 2004-2010 by intended mode of delivery

\begin{tabular}{|c|c|c|c|c|}
\hline Characteristics & $\mathbf{N}$ & Total population & Intended CD* & Intended VD* \\
\hline Deliveries, \% (n) & & $100(450856)$ & $10(45053)$ & $90(405803)$ \\
\hline \multicolumn{5}{|l|}{ Maternal covariates } \\
\hline Smoking & 429008 & $12.6(53905)$ & $11.8(4832)$ & $12.6(49073)$ \\
\hline Pregestational $\mathrm{BMI} \geq 25$ & 418674 & $26.5(111012)$ & $32.1(13715)$ & $25.9(97297)$ \\
\hline Elementary school/high school & & $55.3(249451)$ & $52.0(23447)$ & $55.7(226004)$ \\
\hline Short education/skilled worker & & $6.1(27285)$ & $5.6(2524)$ & $6.1(24761)$ \\
\hline Medium/long education & & $33.6(151415)$ & $34.1(15383)$ & $33.5(136032)$ \\
\hline Previous CD & 441165 & $11.9(52475)$ & $49.4(21741)$ & $7.7(30734)$ \\
\hline \multicolumn{5}{|l|}{ Perinatal covariates } \\
\hline Delivery $<37$ weeks gestation & 450856 & $6.6(29850)$ & $9.2(4155)$ & $6.3(25695)$ \\
\hline PPROM $^{*}$ & 450856 & $6.1(27470)$ & $2.4(1080)$ & $6.5(25390)$ \\
\hline Pre-eclampsia* & 450856 & $4.4(17037)$ & $3.9(1747)$ & $3.8(15290)$ \\
\hline Parous & 444405 & $54.8(247314)$ & $67.5(30421)$ & $53.4(216893)$ \\
\hline Multiple gestation & 450856 & $4.3(19487)$ & $12.5(5621)$ & $3.4(13866)$ \\
\hline Birth weight $<2500 \mathrm{~g}$ & 450856 & $5.2(23267)$ & $8.3(3747)$ & 4.9 (19520) \\
\hline
\end{tabular}

For continuous variables, values are reported as mean \pm 2 SD or as median, 25th and 75 th percentile if not normally distributed. For grouped variables values are reported as $\%(n)$.

*See online supplementary appendix.

BMI, body mass index, CD, caesarean delivery; GDM, gestational diabetes mellitus; PPROM, preterm prelabour rupture of membranes; VD, vaginal delivery. 


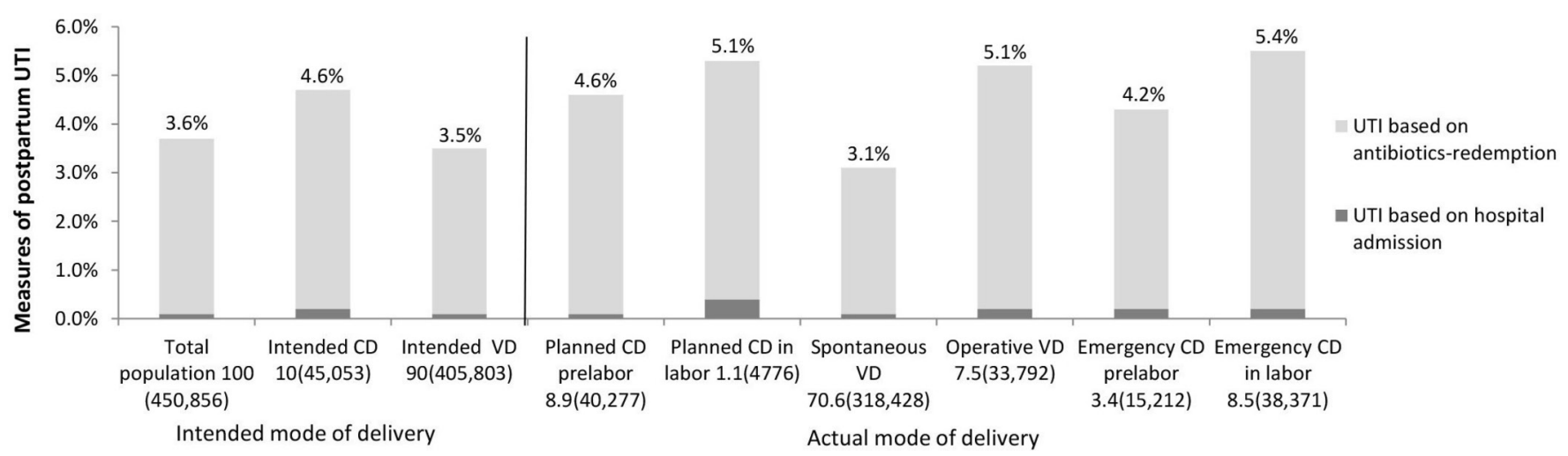

Figure 1 Measures of urinary tract infection (UTI) within 30 days post partum, by mode of delivery. Each bar represents a mode of delivery group, with number of women given as \% (n). Each bar consists of UTI based on admission to hospital (dark grey) and antibiotic prescription redemption (light grey). The total percentage of postpartum UTI of each group is listed above each bar. For definition of mode of delivery, see online supplementary appendix. CD, caesarean delivery; VD, vaginal delivery.

Table 2 Risk of postpartum urinary tract infection within the first 30 days by intended mode of delivery

\begin{tabular}{|c|c|c|c|}
\hline & \multirow{2}{*}{$\begin{array}{l}\text { Crude } \\
\text { OR }(95 \% \mathrm{Cl})\end{array}$} & \multirow{2}{*}{$\begin{array}{l}\text { Adjusted 1* } \\
\text { Adjusted OR (aOR) } \\
(95 \% \mathrm{Cl})\end{array}$} & \multirow{2}{*}{$\begin{array}{l}\text { Adjusted 2† } \\
\text { aOR (95\% Cl) }\end{array}$} \\
\hline & & & \\
\hline No of deliveries & 450856 & 379755 & 379755 \\
\hline No of observed events & 16294 & 13828 & 13828 \\
\hline \multicolumn{4}{|l|}{ Intended mode of delivery } \\
\hline Intended VD $\ddagger$ & 1.0 (reference) & 1.0 (reference) & 1.0 (reference) \\
\hline Intended CD $\ddagger$ & 1.33 (1.27 to 1.40$)$ & 1.24 (1.17 to 1.32$)$ & 1.36 (1.27 to 1.46$)$ \\
\hline \multicolumn{4}{|l|}{ Covariates } \\
\hline Age at delivery & - & 1.00 (0.99 to 1.00$)$ & 1.00 (0.99 to 1.00$)$ \\
\hline Smoking & - & 1.01 (0.96 to 1.06$)$ & 1.01 (0.96 to 1.06$)$ \\
\hline $\mathrm{BMI} \geq 25$ & - & 1.15 (1.10 to 1.19$)$ & 1.15 (1.10 to 1.19$)$ \\
\hline Educational level & - & & \\
\hline Elementary school/high school & - & Reference & Reference \\
\hline Short education/skilled worker & - & 1.01 (0.94 to 1.08$)$ & 1.01 (0.94 to 1.08$)$ \\
\hline Medium/long education & - & 1.00 (0.97 to 1.04$)$ & 1.00 (0.97 to 1.04$)$ \\
\hline GDM $\ddagger$ & - & 1.06 (0.94 to 1.18$)$ & 1.06 (0.95 to 1.18$)$ \\
\hline Infection during pregnancy & - & 2.18 (2.06 to 2.31$)$ & 2.18 (2.06 to 2.30$)$ \\
\hline Birth weight <2500 g & - & 0.83 (0.74 to 0.93$)$ & 0.82 (0.74 to 0.92$)$ \\
\hline Birth $<37$ weeks gestation & - & 0.84 (0.76 to 0.92$)$ & 0.84 (0.76 to 0.92$)$ \\
\hline PPROM & - & 1.09 (1.01 to 1.17$)$ & 1.09 (1.01 to 1.17$)$ \\
\hline Pre-eclampsiał & - & 1.36 (1.26 to 1.47$)$ & 1.36 (1.26 to 1.47$)$ \\
\hline Parous & - & $0.68(0.65$ to 0.71$)$ & 0.68 (0.66 to 0.71$)$ \\
\hline Previous CD & - & 1.53 (1.44 to 1.62$)$ & 1.64 (1.53 to 1.75$)$ \\
\hline $\begin{array}{l}\text { Interaction of previous CD and } \\
\text { intended mode of delivery }\end{array}$ & - & - & 0.79 (0.70 to 0.88$)$ \\
\hline
\end{tabular}

${ }^{*}$ Adjusted for age at delivery, smoking, BMI, education level, GDM, infection during pregnancy, low birth weight, preterm birth, PPROM, preeclampsia, parity and previous CD.

†Adjusted for the variables above and the interaction between previous CD and mode of delivery.

$\ddagger$ See online supplementary appendix.

BMI, body mass index; CD, caesarean delivery; GDM, gestational diabetes mellitus; PPROM, preterm prelabour rupture of membranes; VD, vaginal delivery. 
The covariates showed that infection during pregnancy, PPROM and pre-eclampsia were associated with a significantly higher risk of postpartum UTI, whereas birth weight below $2500 \mathrm{~g}$, preterm delivery and parity were associated with a decreased risk of postpartum UTI.

\section{Risk of postpartum UTI by actual mode of delivery}

Considering actual mode of delivery, women with an emergency caesarean delivery during labour had the highest rate of postpartum UTI $(5.4 \%)$ and women with spontaneous vaginal delivery the lowest $(3.1 \%)$ (figure 1$)$. Crude as well as adjusted logistic regression analyses showed a significantly increased risk of postpartum UTI after operative vaginal delivery (aOR $1.47,95 \%$ CI 1.38 to 1.56 ), planned caesarean delivery prelabour (aOR 1.50, 95\% CI 1.39 to 1.62), planned caesarean delivery in labour (aOR 1.73, 95\% CI 1.44 to 2.08 ), emergency caesarean delivery prelabour (aOR $1.41,95 \%$ CI 1.26 to 1.58 ) and emergency caesarean delivery during labour (aOR 1.61, 95\% CI 1.52 to 1.72) compared with spontaneous vaginal delivery (online supplementary table 2).

\section{The effect of previous caesarean delivery on the risk of} postpartum UTI

As previous caesarean delivery interacted with the association between mode of delivery and postpartum UTI (table 2 and online supplementary table 2, adjusted model 2), data were analysed in stratified analyses (table 3).

Among women without previous caesarean delivery, the risk of postpartum UTI was increased in women with intended caesarean delivery compared with intended vaginal delivery (aOR $1.36,95 \%$ CI 1.26 to 1.46 ), as well as in women giving birth by operative vaginal delivery or caesarean delivery when compared with spontaneous vaginal delivery (table 3 ), and risk estimates did not differ substantially from those in the total population (table 2 and online supplementary table 2).

Among women with a previous caesarean delivery, intended caesarean delivery was not associated with increased risk of postpartum UTI (aOR 1.08, 95\% CI 0.98 to 1.18 ), and increased risk of postpartum UTI was only seen in women after operative vaginal delivery and planned caesarean delivery prelabour (table 3 ).

\section{Timing of postpartum UTI}

The timing of the postpartum UTI diagnosis did not vary by mode of delivery because $75 \%$ of the postpartum UTIs occurred within 15 days post partum, irrespective of mode of delivery (figure 2).

When defining the postpartum period as a 14-day period or as a 45-day period, we found an equally increased risk of postpartum UTI among intended caesarean delivery compared with intended vaginal delivery in the adjusted analyses (aOR 1.35, 95\% CI 1.26 to 1.45 and aOR 1.45, $95 \%$ CI 1.28 to 1.65 , respectively).

\section{Sensitivity analyses}

In sensitivity analyses in which we excluded UTI in women who had redeemed a prescription for dicloxacillin, benzylpenicillin or metronidazole at any time within the first 30 days post partum, we found that $4.2 \%$ of women with intended caesarean delivery and $3.2 \%$ of women with intended vaginal delivery had a postpartum UTI. Also with this definition of UTI, intended caesarean delivery was associated with a significantly higher risk of postpartum UTI than intended vaginal delivery (aOR $1.42,95 \%$ CI 1.23 to 1.62 ).

Table 3 Stratified analyses, showing the risk of postpartum urinary tract infection (UTI) within the first 30 days post partum for women without or with a previous $\mathrm{CD}$, by intended or actual mode of delivery

\begin{tabular}{|c|c|c|c|c|}
\hline \multirow{2}{*}{$\begin{array}{l}\text { Variables } \\
\text { Mode of delivery }\end{array}$} & \multicolumn{2}{|c|}{ Women without previous CD } & \multicolumn{2}{|l|}{ Women with previous CD } \\
\hline & $\begin{array}{l}\text { Prevalence of } \\
\text { postpartum UTI, \% (n) }\end{array}$ & $\begin{array}{l}\text { Adjusted } 1^{*} \text { aOR } \\
(95 \% \mathrm{Cl})\end{array}$ & $\begin{array}{l}\text { Prevalence of } \\
\text { postpartum UTI, \% (n) }\end{array}$ & $\begin{array}{l}\text { Adjusted } 1^{*} \text { aOR } \\
(95 \% \mathrm{Cl})\end{array}$ \\
\hline \multicolumn{5}{|l|}{ Intended mode of delivery† } \\
\hline Intended VD & $3.4(12520)$ & Reference & $4.6(1403)$ & Reference \\
\hline Intended CD & $4.6(1014)$ & 1.36 (1.26 to 1.46$)$ & $4.7(1017)$ & $1.08(0.98$ to 1.18$)$ \\
\hline \multicolumn{5}{|l|}{ Actual mode of delivery $\dagger$} \\
\hline Spontaneous VD† & 3.0 (8919) & Reference & $4.3(688)$ & Reference \\
\hline Operative VD $†$ & $4.9(1524)$ & $1.46(1.37$ to 1.55$)$ & $6.7(163)$ & $1.50(1.24$ to 1.81$)$ \\
\hline Planned CD prelabour† & $4.5(863)$ & 1.49 (1.38 to 1.61$)$ & $4.7(935)$ & $1.14(1.02$ to 1.28$)$ \\
\hline Planned CD in labourt & $5.1(151)$ & 1.72 (1.43 to 2.06$)$ & $4.8(82)$ & $1.13(0.83$ to 1.54$)$ \\
\hline Emergency CD prelabourt & $4.3(433)$ & 1.40 (1.25 to 1.57$)$ & $3.9(183)$ & $1.04(0.84$ to 1.27$)$ \\
\hline Emergency CD in labourt & 5.5 (1644) & $1.60(1.51$ to 1.70$)$ & $4.8(369)$ & $1.06(0.91$ to 1.23$)$ \\
\hline
\end{tabular}

${ }^{*}$ Adjusted for age at delivery, smoking, body mass index, educational level, gestational diabetes mellitus, infection during pregnancy, low birth weight, preterm birth, preterm prelabour rupture of membranes, pre-eclampsia and parity.

†See online supplementary appendix.

aOR, adjusted OR; CD, caesarean delivery; VD, vaginal delivery. 


\section{DISCUSSION}

We found that intended caesarean delivery was associated with a $36 \%$ increased risk of postpartum UTI as compared with intended vaginal delivery. Using actual mode of delivery as exposure, operative vaginal delivery, emergency and planned caesarean delivery carried an equally increased risk of UTI compared with spontaneous vaginal delivery. Intended caesarean delivery was not associated with increased risk of postpartum UTI in women with a previous caesarean section.

\section{Strengths and limitations}

This study is the first on postpartum UTI to mimic an intention-to-treat analyses and to include operative vaginal delivery as a category of actual mode of delivery. The positive association between mode of delivery and postpartum UTI may be attributed to pregnancy, general health or lifestyle differences, making residual or unknown confounding possible. We did, however, adjust for a number of known confounding factors.

This cohort study was population based. It is a strength that the Danish registries provide nationwide, almost complete data with high validity, and the prospective collection limits the risk of selection and information bias. ${ }^{13}$ More than $99 \%$ of Danish women deliver in hospitals, ${ }^{18}$ and health service in Denmark is free of charge. We demonstrated that very few postpartum UTIs require hospital admission, indicating that most postpartum UTIs are mild. A limitation of our study was that the diagnosis of postpartum UTI was not confirmed by information regarding urinary cultures. Nevertheless, we found an incidence of postpartum UTI in accordance with that of other studies, ${ }^{17}$ also those including information on urine cultures. ${ }^{36}$ Danish recommendations discourage treatment of UTI with antibiotics before a biochemical test confirms the diagnosis, and most general practitioners use dipsticks, microscopic examination and/or cultures to confirm the diagnoses, but these data are not reported to the Danish registers. Treatment initiation before the final test results would introduce an increased prevalence of postpartum UTI in our data. However, we do not suspect this approach to differ between delivery groups because the timing of postpartum UTI was similar in all groups.

Endometritis and wound infection could be mistaken for a UTI in the early postpartum phase. As these infections occur more often among women having had emergency caesarean delivery, ${ }^{319}$ who in our study were included in the reference group, it would favour rejection of the null hypothesis. Using a more restrictive definition of postpartum UTI in our sensitivity analyses did not essentially change the estimates, which indicates a high robustness of the results. Finally, postpartum discomfort after caesarean delivery could be mistaken for a UTI, ${ }^{20} 21$ but, as mentioned above, we found no indication that the timing of postpartum UTI was affected by actual mode of delivery.

In this study, we found that low birth weight, preterm delivery and parity were associated with a decreased risk of postpartum UTI. These deliveries are more likely to proceed faster, with less risk of operative intervention and potentially altered hormonal influences, which could explain the lower risk of UTI associated with these deliveries.

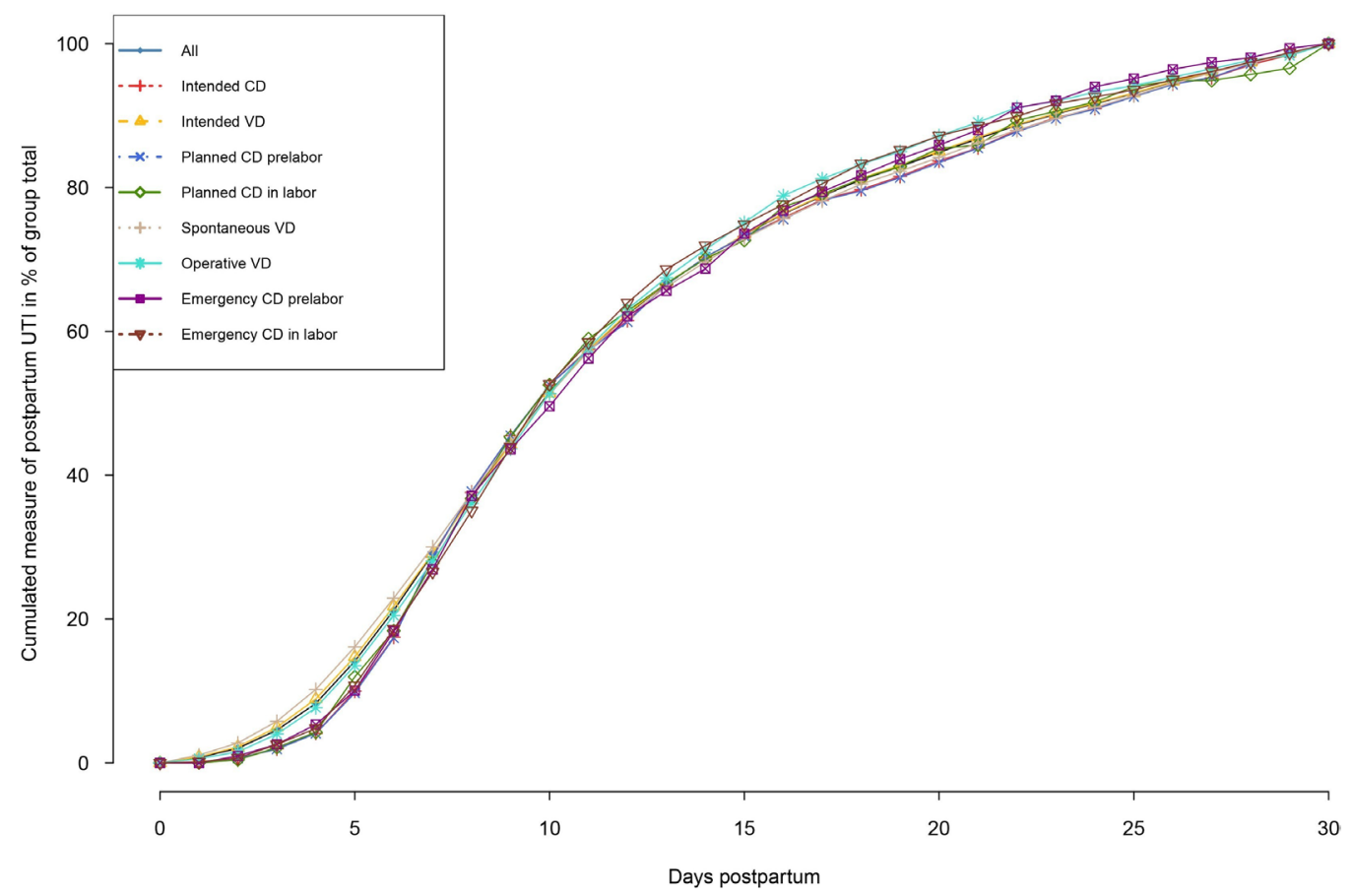

Figure 2 Cumulated incidence of urinary tract infection (UTI) by days post partum. Each line represents a mode of delivery group. The cumulated incidence is shown as the percentage of all UTIs per mode of delivery. For definition of mode of delivery, see online supplementary appendix. CD, caesarean delivery; VD, vaginal delivery. 


\section{Other studies}

We found that $3.6 \%$ of all women were treated for postpartum UTI, which is in accordance with other Scandinavian studies reporting incidences of postpartum UTI of $2 \%-3 \% .{ }^{1617}$ There are no studies on intended mode of delivery for comparison. In studies using actual mode of delivery as exposure, caesarean delivery was associated with an increased risk of postpartum UTI compared with vaginal delivery. ${ }^{5-7}$ One small study found an increased risk of postpartum UTI after emergency caesarean delivery compared with planned caesarean delivery. ${ }^{22}$ This is in accordance with the crude estimates in our study, as the authors did not adjust for confounders. Other studies found no difference in the risk of postpartum UTI between planned and emergency caesarean delivery after adjusting for relevant confounders, ${ }^{6} 1923$ which our findings further support. Previous caesarean delivery has been related to an increased risk of postpartum complications, ${ }^{2425}$ but no prior study has evaluated the influence of previous caesarean delivery on postpartum UTI. We found an interaction between previous caesarean delivery and mode of delivery with regard to the risk of postpartum UTI, as intended caesarean delivery was not associated with increased risk of postpartum UTI in women with a previous caesarean delivery. This could be explained by a higher prevalence of emergency caesarean section and a higher prevalence of postpartum UTI after vaginal delivery in the group (table 3 ).

In this study all women with operative delivery, whether caesarean or operative vaginal delivery, had an increased risk of postpartum UTI. A possible explanation could be that national and international guidelines recommend sterile urinary bladder catheterisation of women before caesarean delivery and operative vaginal delivery either by intermittent catheterisation or as a catheter a demeure. ${ }^{26}{ }^{27}$ The purpose of bladder catheterisation is to prevent surgical damage to a distended bladder during the procedure and to prevent postpartum haemorrhage as well as urinary retention due to lack of bladder function caused by the spinal anaesthesia. To decrease the risk of UTI, it is standard procedure to remove the catheter as soon as the woman is mobilised. Emptying the bladder prior to operative vaginal delivery is thought to facilitate the descent of the fetal head into the maternal pelvis, however, previous studies suggest that indwelling catheterisation during caesarean delivery increases the risk of postpartum UTI compared with non-use. ${ }^{28} 29$ The catheterisation procedure itself and not the delivery mode could explain the risk of postpartum UTI: a hypothesis that is further supported by the similar risk of postpartum UTI across all our operative delivery groups. Therefore, the risk of postpartum UTI must be counterbalanced by the potential benefits associated with catheterisation prior to caesarean delivery and operative vaginal delivery.

This study provides relevant risk estimates to clinicians, when guiding pregnant women in determining mode of delivery. UTI is a minor complication, and though the risk is increased in women having caesarean delivery compared with women giving birth vaginally the absolute risk in both groups is rather small. Therefore, the increased risk of UTI potentially caused by urine bladder catheterisation seems to be justified by the potential prevention of more serious complications. However, regarding women aiming for caesarean on maternal request, both major and minor complications should be taken into consideration, in order to reduce the rate of caesarean deliveries without medical indication. Regarding routine use of urine bladder catheterisation prior to operative vaginal delivery, studies to evaluate bladder catheterisation procedures as well as other procedures, like bedside ultrasound, to measure urine bladder volume prior to catheterisation should be encouraged.

In conclusion, intended caesarean delivery was associated with a small increase in the risk of postpartum UTI as compared with intended vaginal delivery, and all types of operative delivery were associated with an equally increased risk of postpartum UTI compared with spontaneous vaginal delivery. The frequent use of urinary bladder catheterisation prior to surgical procedures could explain this association. The minor risk of UTI associated with operative delivery should be held against the potential prevention of more serious complications like bladder lesions and postpartum haemorrhage. Future studies should focus on reducing routine catheterisation prior to operative vaginal delivery as well as improving procedures related to catheterisation prior to operative delivery.

Contributors TDG, TDC, ECLL, SCR, JG and LK conceived the study. TDG, SCR and TDC were involved in collection and analysis of the data. TDG wrote the initial draft. TDC, ECLL, LK, JG and SCR provided feedback and comments. All authors approved the final version of the manuscript.

Funding This study was funded by The Danish Council for Independent Research, grant number DFF-6110-00283.

Disclaimer The funding source had no involvement in study design, collection, analyses or interpretation of data in the writing of the report or the decision to submit the article for publication.

Competing interests None declared.

Patient consent Not required.

Ethics approval The study is in accordance with Danish regulations and included only deidentified data from national registries. The Danish Data Protection Agency approved the study (journal number: 2012-58-0004, NOH-2016-004).

Provenance and peer review Not commissioned; externally peer reviewed.

Data sharing statement This study was conducted using official data from Danish registries. Accesses to the Danish national registries are provided by Statistics Denmark. Data can be accessed for research by applying for specific dataset extraction. No additional unpublished data from the study are publicly available.

Open Access This is an Open Access article distributed in accordance with the Creative Commons Attribution Non Commercial (CC BY-NC 4.0) license, which permits others to distribute, remix, adapt, build upon this work non-commercially, and license their derivative works on different terms, provided the original work is properly cited and the use is non-commercial. See: http://creativecommons.org/ licenses/by-nc/4.0/

(c) Article author(s) (or their employer(s) unless otherwise stated in the text of the article) 2018. All rights reserved. No commercial use is permitted unless otherwise expressly granted. 


\section{REFERENCES}

1. Ahnfeldt-Mollerup P, Petersen LK, Kragstrup J, et al. Postpartum infections: occurrence, healthcare contacts and association with breastfeeding. Acta Obstet Gynecol Scand 2012;91:1440-4.

2. Couto RC, Pedrosa TM, Nogueira JM, et al. Post-discharge surveillance and infection rates in obstetric patients. Int J Gynaecol Obstet 1998;61:227-31.

3. Leth RA, Nørgaard M, Uldbjerg N, et al. Surveillance of selected post-caesarean infections based on electronic registries: validation study including post-discharge infections. J Hosp Infect 2010;75:200-4.

4. Jaiyeoba O. Postoperative infections in obstetrics and gynecology. Clin Obstet Gynecol 2012;55:904-13.

5. Yokoe DS, Christiansen CL, Johnson R, et al. Epidemiology of and surveillance for postpartum infections. Emerg Infect Dis 2001;7:837-41.

6. Leth RA, Møller JK, Thomsen RW, et al. Risk of selected postpartum infections after cesarean section compared with vaginal birth: a five-year cohort study of 32,468 women. Acta Obstet Gynecol Scand 2009;88:976-83.

7. Hung HW, Yang PY, Yan YH, et al. Increased postpartum maternal complications after cesarean section compared with vaginal delivery in 225304 Taiwanese women. J Matern Fetal Neonatal Med 2016;29:1-8.

8. NICE. Caesarean section guidelines: NICE Clin Guidel [Internet], 2011. http://www.nice.org.uk/nicemedia/live/13620/57162/57162. pdf.

9. Knudsen LB, Olsen J. The Danish medical birth registry. Dan Med Bull 1998;45:320-3.

10. Danmarks Statistik. Fertilitetsdatabasen [Internet]. http://www.dst.dk/ $\mathrm{da} /$ Statistik/dokumentation/Times/fertilitetsdatabasen\# (accessed 13 Sep 2015).

11. Andersen TF, Madsen M, Jørgensen J, et al. The Danish National Hospital Register. A valuable source of data for modern health sciences. Dan Med Bull 1999;46:263-8.

12. Kildemoes HW, Sørensen HT, Hallas J. The Danish National Prescription Registry. Scand J Public Health 2011;39(7 Suppl):38-41.

13. Pedersen CB. The Danish civil registration system. Scand J Public Health 2011;39(7 Suppl):22-5.

14. Arendrup K, Arpi M, Jakobsen HN, et al; Antibiotikavejledning i almen praksis - Region H, 2016:1-20.
15. Hovedstaden R. Forholdsregler Og Behandlingsrekommandationer Håndbog, 2015.

16. Nordisk Medicinal-Statistisk Komité. NOMESCO classification of surgical procedures. Copenhagen [Albertslund]: Nordic MedicoStatistical Committee (NOMESCO), 2007.

17. Axelsson D, Blomberg M. Prevalence of postpartum infections: a population-based observational study. Acta Obstet Gynecol Scand 2014;93:1065-8.

18. Sundhedsstyrelsen. Hjemmefødsel [Internet]: Sundhed.dk, 2012:1. https://www.sundhed.dk/borger/sundhed-og-forebyggelse/ graviditet-foedsel-barsel/foedsel-liste/foedesteder/hjemmefoedsel/

19. Hillan EM. Postoperative morbidity following Caesarean delivery. $J$ Adv Nurs 1995;22:1035-42.

20. Rowlands IJ, Redshaw M, Saurel-Cubizolles M. Mode of birth and women's psychological and physical wellbeing in the postnatal period. BMC Pregnancy Childbirth 2012;12:138.

21. Schind $M$, Birner $P$, Reingrabner $M$, et al. Elective cesarean section vs. spontaneous delivery: a comparative study of birth experience. Acta Obstet Gynecol Scand 2003;82:834-40.

22. Suwal A, Shrivastava VR, Giri A. Maternal and fetal outcome in elective versus emergency cesarean section. JNMA J Nepal Med Assoc 2013;52:563-6.

23. Parrott T, Evans AJ, Dennis KJ. Infection following section. Infection 1989:349-54.

24. American College of Obstetricians and Gynecologists. ACOG Practice bulletin no. 115: Vaginal birth after previous cesarean delivery. Obstet Gynecol 2010;116(2 Pt 1):450-63.

25. Gupta J, Smith G, Chodankar R. Executive summary of recommendations Antenatal care schedule: Green-top Guidel, 2015:45. (Birth After Previous Caesarean Birth).

26. Bahl DR, Strachan BK, Murphy DJ. Operative vaginal delivery: Green-top Guidel, 2011:26.

27. Committee on Practice Bulletins-Obstetrics. ACOG practice bulletin No. 154: operative vaginal delivery. Obstet Gynecol 2015;126:e56-65.

28. Li L, Wen J, Wang L, et al. Is routine indwelling catheterisation of the bladder for caesarean section necessary? A systematic review. BJOG 2011;118:400-9.

29. Schwartz MA, Wang CC, Eckert LO, et al. Risk factors for urinary tract infection in the postpartum period. Am J Obstet Gynecol 1999;181:547-53. 